\title{
Numerical Method in Two Dimensional Boundary Layer Theory for Incompressible Viscous Fluid
}

\author{
M. A. Haque \\ Department of Applied Mathematics \\ University of Rajshahi, Bangladesh
}

\begin{abstract}
In this paper laminar flow of incompressible viscous fluid has been considered. Here two numerical methods for solving boundary layer equation have been discussed; (i) Keller Box scheme, (ii) Shooting Method. In Shooting Method, the boundary value problem has been converted into an equivalent initial value problem. Finally the RungeKutta method is used to solve the initial value problem.
\end{abstract}

Keyword: Box scheme, shooting method, tri-diagonal matrix, Runge-Kutta method, incompressible viscous fluid

\section{Introduction}

In many flow problems the partial differential equations governing the motion of the fluid are nonlinear. These partial nonlinear equations cannot be solved easily. Some suitable transformations of variables can reduce the system of partial differential equations to a system of ordinary differential equations. An important contribution to fluid dynamics was made by L Prandtl in 1904 by introducing the concept of boundary layer. According to him if a slightly viscous fluid flows over a body such that the Reynold number is very large, then there is a thin layer near the body where the viscous forces are important; and outside the layer the viscous forces are unimportant. There is a variety of numerical methods that are used to solve the many flow problems. Two particular methods, the Crank Nicolson Scheme and the Keller Box scheme, seem to dominate in most practical applications. Of these Box scheme method is easy to adapt to new classes of problems. The Box scheme was devised by Keller [3] for solving diffusion problems and applied by him to a variety of boundary layer flow problems $[2,3]$. Here we have engaged in numerical simulation of fluid dynamics using computer. Here we have modified the Blasius equation into the equivalent first order system of equations. To find out the numerical solution of the problem we have used the shooting method and Runge-Kutta method. 


\section{Mathematical formulation}

For steady laminar flow of incompressible viscous fluid the basic equation of mass conservation and momentum are :

$$
\frac{\partial u_{i}}{\partial x_{i}}=0
$$

and $\quad \frac{\partial u_{i}}{\partial t}+u_{j} \frac{\partial u_{i}}{\partial x_{j}}=-\frac{1}{\rho} \frac{\partial p}{\partial x_{i}}+v \nabla^{2} u_{i}$

Suppose the fluid flows in the $\mathrm{x}$ direction only and varies perpendicularly to the $x$ axis(i.e. $y$ direction). According to the concepts of Prandtl, we obtain the following equations for two dimensional boundary layer flow:

$$
\begin{aligned}
& \frac{\partial u}{\partial t}+u \frac{\partial u}{\partial x}+v \frac{\partial u}{\partial y}=-\frac{1}{\rho} \frac{\partial p}{\partial x}+v \frac{\partial^{2} u}{\partial y^{2}} \\
& \frac{\partial p}{\partial y}=0 \\
& \frac{\partial u}{\partial x}+\frac{\partial v}{\partial y}=0
\end{aligned}
$$

with boundary conditions

$$
\begin{aligned}
& \mathrm{u}=0, \mathrm{v}=0 \text { when } \mathrm{y}=0 \\
& u=\bigcup_{\infty}(x, t) \text { when } y \rightarrow \infty
\end{aligned}
$$

\section{A: Tri-diagonal system}

An efficient technique sometimes called Thomas algorithm can be used to solve a linear system with a tri-diagonal matrix defined by the following equations

$$
\begin{aligned}
& b_{1} u_{1}+c_{1} u_{2}=d_{1} \\
& a_{2} u_{1}+b_{2} u_{2}+c_{2} u_{3}=d_{2} \\
& a_{3} u_{2}+b_{3} u_{3}+c_{3} u_{4}=d_{3} \\
& \ldots \ldots \ldots \ldots \ldots \ldots \ldots \ldots \ldots \ldots \ldots \ldots \ldots \ldots \\
& \ldots \ldots \ldots \ldots \ldots \ldots \\
& a_{n} u_{n-1}+b_{n} u_{n}=d_{n}
\end{aligned}
$$

where $u_{i}$ are the unknowns and $a_{i}, b_{i}, c_{i}, d_{i}$ are known.

It is possible to calculate the unknown ${ }^{u_{i}}$ using the following recurrence formula 


$$
u_{i}=\beta_{i}-\frac{c_{i} u_{i+1}}{\alpha_{i}}, \quad i=n-1, n-2, \ldots \ldots \ldots \ldots \ldots \ldots \ldots \ldots \ldots \ldots \ldots
$$

where $u_{n}=\beta_{n}$

Setting $\alpha_{1}=b_{1}$ and calculating

$$
\alpha_{i}=b_{i}-\frac{a_{1} c_{i-1}}{\alpha_{i-1}}, \quad i=2,3,, \ldots \ldots \ldots \ldots \ldots \ldots n
$$

Again setting $\beta_{1}=\frac{d_{1}}{b_{1}}$ and calculating

$$
\beta_{i}=\frac{d_{i}-a_{i} \beta_{i-1}}{\alpha_{i}}, \quad i=2,3, \ldots \ldots \ldots \ldots \ldots \ldots \ldots, n
$$

Finally the recurrence formula (8) is used to calculate all $u_{i}$ successively for $i=n-1, n-2, \ldots \ldots . .1$.

\section{B: Box Scheme}

Let $\tau$ be the shear stress of the flowing fluid whose viscosity is $\mu$.For Newtonian fluid, we have

$$
\tau=\mu \frac{\partial u}{\partial y}
$$

Eq.(3), Eq.(4), Eq.(5) and Eq.(11) yield the following equations:

$$
\begin{aligned}
& u \frac{\partial u}{\partial x}+v \frac{\partial u}{\partial y}=-\frac{1}{\rho} \frac{d p}{d x}+\frac{1}{\rho} \frac{\partial \tau}{\partial y} \\
& \frac{\partial u}{\partial x}+\frac{\partial v}{\partial y}=0
\end{aligned}
$$

Boundary conditions:

$$
\begin{aligned}
& u(x, 0)=0 \\
& v(x, 0)=0 \\
& u\left[x, y_{\delta}(x)\right]=U_{\infty}
\end{aligned}
$$


where $U_{\infty}$ represents the free stream velocity, $\delta$ the boundary layer thickness.

We have imposed the following additional condition:

$$
\partial u\left[x, y_{\delta}(x)\right] / \partial y=0
$$

Outside the boundary layer, we have

$$
U_{\infty} \frac{d U_{\infty}}{d x}=-\frac{1}{\rho} \frac{d p}{d x}
$$

We now introduce the following transformations:

$$
\begin{aligned}
& \eta(x, y)=y\left[U_{\infty}(x) / \mid v_{0} x\right]^{1 / 2} \\
& f(x, \eta)=\psi(x, y) \mid\left[U_{\infty}(x) v_{0} x\right]^{1 / 2} \\
& P(x)=\frac{x}{U_{\infty}(x)} \frac{d U_{\infty}(x)}{d x} \\
& b(x, \eta)=\frac{v(x, y)}{v_{o}}
\end{aligned}
$$

Here $v_{o}$ is some reference kinematic coefficient of viscosity and $\psi$ is the stream function for which

$$
\begin{aligned}
& u(x, y)=\frac{\partial \psi}{\partial y} \\
& v(x, y)=-\frac{\partial \psi}{\partial x}
\end{aligned}
$$

Using Eq. (16) and Eq. (17) in Eq. (12), we get

$$
\frac{\partial}{\partial \eta}\left(b \frac{\partial^{2} f}{\partial \eta^{2}}\right)+\frac{1}{2}(P+1) f \frac{\partial^{2} f}{\partial \eta^{2}}+P\left[1-\left(\frac{\partial f}{\partial \eta}\right)^{2}\right]=x\left[\frac{\partial f}{\partial \eta} \frac{\partial^{2 f}}{\partial x \partial \eta}-\frac{\partial^{2} f}{\partial \eta^{2}} \frac{\partial f}{\partial x}\right]
$$

Eq. (18) is equivalent to the following first order system of equations:

$$
\frac{\partial f}{\partial \eta}=U
$$




$$
\begin{aligned}
& \frac{\partial U}{\partial \eta}=V \\
& \frac{\partial}{\partial \eta}(b V)=x\left[U \frac{\partial U}{\partial x}-V \frac{\partial f}{\partial x}\right]-\frac{P+1}{2} f V-P\left[1-U^{2}\right]
\end{aligned}
$$

The boundary conditions (14) becomes

$$
\begin{aligned}
& f(x, 0)=0 \\
& U(x, 0)=0 \\
& U\left(x, \eta_{\delta}(x)\right)=1
\end{aligned}
$$

Let the x-y plane be divided into a network of rectangles of sides $k_{n}$

$(n=1,2, \ldots \ldots \mathrm{N})$ and $h_{j}(j=1,2,3, \ldots \ldots . . J)$ by drawing the sets lines

$$
\begin{array}{ll}
x_{0}=0, & x_{n}=x_{n-1}+k_{n}, \quad 1 \leq n \leq N ; \\
y_{0}=0 & y_{j}=y_{j-1}+h_{j} \quad 1 \leq j \leq J, y_{J}=y_{\delta}
\end{array}
$$

With the help of Eq. (21) we draw the following nets, some points of intersection of these families of lines are shown by following circles indicated in Fig-1 ..

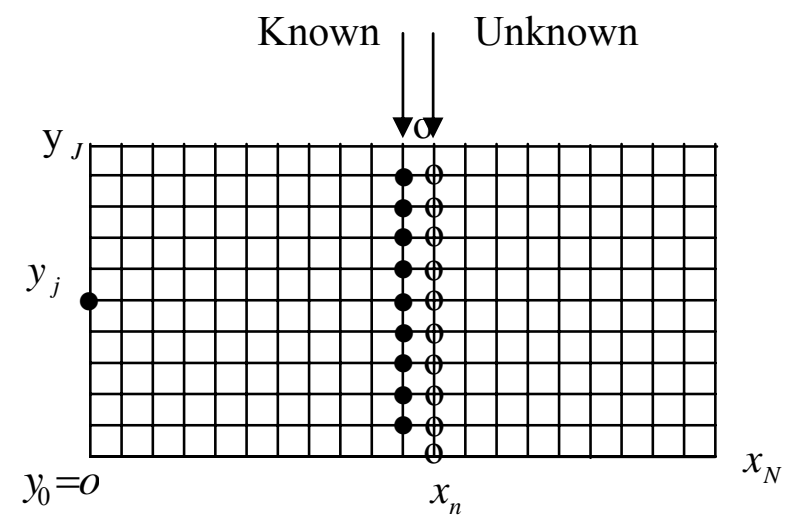

Fig-1 .. 
Now we draw the above indicated box as:

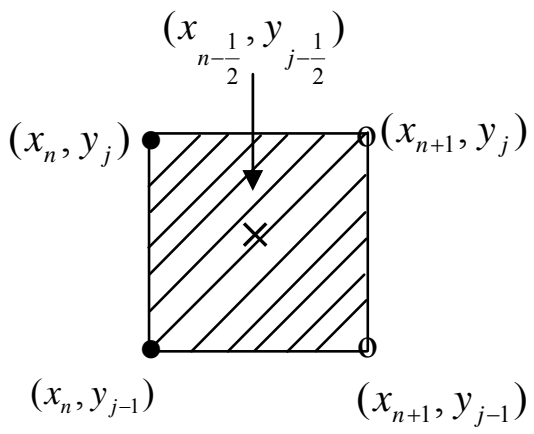

Figure -2

Since the boundary layer equations have been formulated as a first order system, all the derivatives can be approximated by the backward difference approximation for first derivatives and two point averages for dependent variables. Thus any net quantity w may be expressed by the following notations.

$$
\begin{aligned}
& {[w]_{j-\frac{1}{2}}^{n}=\frac{1}{2}\left(w_{j}^{n}+w_{j-1}^{n}\right)} \\
& {\left[\frac{\partial w}{\partial y}\right]_{j-\frac{1}{2}}^{n}=\frac{1}{h_{j}}\left(\left(w_{j}^{n}-w_{j-1}^{n}\right)+o\left(h_{j}\right)\right.} \\
& {\left[\frac{\partial w}{\partial x}\right]_{j-\frac{1}{2}}^{n-\frac{1}{2}}=\frac{1}{k_{n}}\left([w]_{j-\frac{1}{2}}^{n}-[w]_{j-\frac{1}{2}}^{n-1}\right)+o\left(k_{n)}\right.} \\
& {\left[\frac{\partial w}{\partial y}\right]_{j-\frac{1}{2}}^{n-\frac{1}{2}}=\frac{1}{2}\left(\left[\frac{\partial w}{\partial y}\right]_{j-\frac{1}{2}}^{n}+\left[\frac{\partial w}{\partial y}\right]_{j-\frac{1}{2}}^{n-1}\right)}
\end{aligned}
$$




$$
[w]_{j-\frac{1}{2}}^{n-\frac{1}{2}}=\frac{1}{2}\left([w]_{j-\frac{1}{2}}^{n}+[w]_{j-\frac{1}{2}}^{n-1}\right)
$$

It may be mentioned that $\left[\frac{\partial w}{\partial y}\right]_{j-\frac{1}{2}}^{n-\frac{1}{2}}$ is the best difference approximation to

$\partial w\left(x_{n-\frac{1}{2}}, y_{j-\frac{1}{2}}\right) / \partial y$.If we omit local truncation error and apply the Box scheme in Eq.(19) we get

$$
\begin{aligned}
& {\left[\frac{\partial f}{\partial \eta}\right]_{j-\frac{1}{2}}^{n}=[U]_{j-\frac{1}{2}}^{n}} \\
& {\left[\frac{\partial U}{\partial \eta}\right]_{j-\frac{1}{2}}^{n}=[V]_{j-\frac{1}{2}}^{n}} \\
& {\left[\frac{\partial(b V)}{\partial \eta}\right]_{j-\frac{1}{2}}^{n-\frac{1}{2}}=x_{n-\frac{1}{2}}\left\{[U]_{j-\frac{1}{2}}^{n-\frac{1}{2}}\right\}\left[\frac{\partial U}{\partial x}\right]_{j-\frac{1}{2}}^{n-\frac{1}{2}}-[V]_{j-\frac{1}{2}}^{n-\frac{1}{2}}\left[\frac{\partial f}{\partial x}\right]_{j-\frac{1}{2}}^{n-\frac{1}{2}}-\left[\frac{p+1}{2} f V-P\left(1-U^{2}\right)\right]_{j-\frac{1}{2}}^{n-\frac{1}{2}}}
\end{aligned}
$$

For example we use the following identity

$$
[V \tau / v]_{j-\frac{1}{2}}^{n-\frac{1}{2}} \text { or }[V \tau]_{j-\frac{1}{2}}^{n-\frac{1}{2}}[v]_{j-\frac{1}{2}}^{n-\frac{1}{2}} \text { or }[V]_{j-\frac{1}{2}}^{n-1}[\tau]_{j-\frac{1}{2}}^{n-\frac{1}{2}}[V]_{j-\frac{1}{2}}^{n-\frac{1}{2}}
$$

There are numerous other ways in which the last term in Eq.(23.c) could have been written while retaining the proper centering. Keller[2] solved the Eq. (23.c) by using Newton's method. The resulting system of linear algebraic equations has tri-diagonal form but with different elements in the vector and matrices. Using the procedure for solving tri-diagonal system the resulting equation or equivalent system can be easily solved by the Box scheme. 


\section{C: Shooting Method}

Let us consider a thin infinite flat plate submerged in steady two dimensional flow whose undisturbed velocity is $U_{\infty}$. Again suppose that the fluid is incompressible with low viscosity. We will now proceed toward this end by concentrating on getting to the aforementioned ordinary differential equation, which is the well-known Blasius equation. To do this we introduce the stream function $\psi$, such that

$$
\begin{aligned}
& u=\frac{\partial \psi}{\partial y} \\
& v=-\frac{\partial \psi}{\partial x}
\end{aligned}
$$

We introduce the following new variables:

$$
\begin{aligned}
& \eta=y \sqrt{\frac{U_{\infty}}{v x}} \\
& f(\eta)=\frac{\psi(x, y)}{\sqrt{v x U_{\infty}}}
\end{aligned}
$$

Using Eq.(24) in Eq.(3) we get

$$
\begin{aligned}
& 2 f^{\prime \prime \prime}+f f^{\prime \prime}=0 \\
& f(0)=0, \quad f^{\prime}(0)=0, \quad f^{\prime}(\infty)=1
\end{aligned}
$$

Analytic solution:

Blasius obtained the solution of Eq. (25) with the help of (26) in the form of power series expansion about $\eta=0$ assuming the following form:

$$
f(\eta)=A_{0}+A_{1} \eta+\frac{A_{2}}{2 !} \eta^{2}+\frac{A^{3}}{3 !} \eta^{3}+\cdots \cdots \cdots \cdots \cdots
$$

The boundary conditions $f(0)=0$ and $f^{\prime}(0)=0$, reduces

$$
f(\eta)=A_{2}^{1 / 3} F\left(A_{2}^{1 / 3} \eta\right)
$$


The third boundary condition $f^{\prime}(\infty)=1$ yields

$$
A_{2}=\left[\frac{1}{\lim _{\eta \rightarrow \alpha} F^{\prime}(\eta)}\right]^{3 / 2}
$$

The value of $A_{2}$ can be obtained numerically from (29). Howarth found that $\mathrm{A}_{2}=0.332$.

Numerical Solution :

Eq. (25) is equivalent to the following system of equations:

$$
\begin{aligned}
& \frac{d f}{d \eta}=u \\
& \frac{d u}{d \eta}=v \\
& \frac{d v}{d \eta}=-\frac{1}{2} f v
\end{aligned}
$$

The boundary conditions (26) becomes

$$
\begin{aligned}
& f(0)=0 \\
& u(0)=0 \\
& u(\infty)=1
\end{aligned}
$$

If we find out $v(0)$ with the help of $u(\infty)=1$ then Eq.(30) is said to be a initial value problem . To convert Eq. (30) as initial value problem, we may use shooting method. Let $v(0)=M_{1}$. Then the above system can be solved for $f, u$ and $v$ using any initial value method using steps of $h$, until the solution at $\eta=\infty$ is reached. Let $u(\infty)=B_{1}$. If $B_{1}=1$, then we have obtained the required solution. In practices, it is very unlikely that our initial guess $v(0)=M_{1}$ is 
correct. If $B_{1} \neq 1$ then we obtain the solution with another guess, say $v(0)=M_{2}$. Let $u(\infty)=B_{2}$. If $B_{2}$ is not equal to 1 then we can calculate the third approximate value $\bar{v}(0)=M_{3}$ (say) by the following formula:

$$
\bar{v}(0)=M_{2}-\frac{B_{2}-1}{B_{2}-B_{1}} \times\left(M_{2}-M_{1}\right)
$$

We make the same kind calculation as above by using $\bar{v}(0)$ and take the better of the two initial values $v(0)$. In this way we can find another improved value of $v(0)$. This process may be continued. The process is carried out until the change of $v(0)$ at successive computations is less than some small prescribed value $\varepsilon, \mathrm{i}$, e,

$$
\frac{[v(0)]_{k+1}-[v(o)]_{k}}{[v(0)]_{k}}<\varepsilon
$$

After getting the approximate value of $v(0)$ we may represent this initial value problem by following form:

$$
\begin{gathered}
\frac{d \bar{u}}{d \eta}=\bar{f}(\eta, f, u, v) \\
\bar{u}(0)=\bar{\eta}
\end{gathered}
$$

The initial value problem may be solved by fourth order Runge-Kutta method as

$$
\bar{u}_{j+1}=\bar{u}_{j}+\frac{1}{6}\left(\bar{k}_{1}+2 \bar{k}_{2}+2 \bar{k}_{3}+\bar{k}_{4}\right)
$$


where

$$
\begin{array}{cc}
\bar{k}_{1}=\left[\begin{array}{l}
k_{11} \\
k_{21} \\
k_{31}
\end{array}\right] & \bar{k}_{2}=\left[\begin{array}{l}
k_{12} \\
k_{22} \\
k_{32}
\end{array}\right] \\
\bar{k}_{3}=\left[\begin{array}{l}
k_{13} \\
k_{23} \\
k_{33}
\end{array}\right] & \bar{k}_{4}=\left[\begin{array}{l}
k_{14} \\
k_{24} \\
k_{34}
\end{array}\right]
\end{array}
$$

and

$$
\begin{aligned}
& k_{i 1}=h f_{i}\left(\eta_{j}, u_{1 j}, u_{2 j}, u_{3 j}\right) \\
& k_{i 2}=h f_{i}\left(\eta_{j}+\frac{h}{2}, u_{1 j}+\frac{1}{2} k_{11}, u_{2 j}+\frac{1}{2} k_{21}, u_{3 j}+\frac{1}{2} k_{31}\right) \\
& k_{i 3}=h f_{i}\left(\eta_{j}+\frac{h}{2}, u_{1 j}+\frac{1}{2} k_{12}, u_{2 j}+\frac{1}{2} k_{22}, u_{3 j}+\frac{1}{2} k_{32}\right) \\
& k_{i 4}=h f_{i}\left(\eta_{j}+h, u_{1 j}+k_{13}, u_{2 j}+k_{23}, u_{3 j}+k_{33}\right) \\
& \quad i=1,2,3
\end{aligned}
$$

\section{Example:}

$$
\frac{d f}{d \eta}=u
$$

$$
\frac{d u}{d \eta}=v
$$

$$
\frac{d v}{d \eta}=-\frac{1}{2} f v
$$

$$
f(0)=0, \quad u(0)=0, \quad v(2.1)=1, \quad h=.1,
$$


By shooting method we have $\mathrm{v}(0)=.32$

\begin{tabular}{|c|c|c|c|}
\hline$\eta$ & $f$ & $u$ & $v$ \\
\hline 0 & 0 & 0 & 0.32 \\
\hline .1 & 0.0016 & 0.032 & 0.31999 \\
\hline .2 & 0.0064 & 0.06399 & 0.319932 \\
\hline .3 & 0.01439 & 0.09598 & 0.31977 \\
\hline .4 & 0.02559 & 0.12794 & 0.319454 \\
\hline .5 & 0.03998 & 0.15986 & 0.318935 \\
\hline .6 & 0.046634 & 0.172615 & 0.31866 \\
\hline .7 & 0.05379 & 0.185351 & 0.31958 \\
\hline .8 & 0.06145 & 0.19812 & 0.321893 \\
\hline .9 & 0.06963 & 0.21098 & 0.32433 \\
\hline 1.0 & 0.07833 & 0.223945 & 0.32539 \\
\hline 1.1 & 0.13661 & 0.29642 & 0.32144 \\
\hline 1.2 & 0.21101 & 0.36819 & 0.32818 \\
\hline 1.3 & 0.3016 & 0.446702 & 0.348167 \\
\hline 1.4 & 0.41029 & 0.53395 & 0.368238 \\
\hline 1.5 & 0.53895 & 0.62278 & 0.36755 \\
\hline 1.6 & 0.6874 & 0.70244 & 0.34322 \\
\hline 1.7 & 0.85313 & 0.77620 & 0.31490 \\
\hline 1.8 & 1.0346 & 0.84325 & 0.28336 \\
\hline 1.9 & 1.23026 & 0.902962 & 0.24964 \\
\hline 2.0 & 1.4630 & 0.96043 & 0.21105 \\
\hline 2.1 & 1.7090 & 1 & 0.17316 \\
\hline
\end{tabular}




\section{Conclusion}

Complete solution of Navier-Stokes equation is not possible and so the flow region is split up into two regions; the boundary layer region and the potential flow region. To establish the accuracy of the solutions of boundary layer problem the Keller-Box scheme and the shooting method are employed. Numerical computation for shooing method is carried out with the help of Runge-Kutta method. These methods have been employed to find out the velocity components of the flow. This can result in significant savings of time when one can replace the expensive and time consuming experimentation that would otherwise be needed.

\section{References}

1. J. D. Anderson: Jr. et. al..., in J. F. Wendt (ed) Introduction to Computational Fluid Dynamics, Springer Berlin p.p. 170-172 (1992)

2. Keller H. B. : Numerical methods in boundary layer theory, Ann. Rev. Fluid Mech. Vol. 10, 417-33 (1978)

3. Keller H.B: A new difference scheme for parabolic problems. Numerical Solution of Partial Differential Equations, cd. B Hubbard 2: 327-50 Academic press, New York, p. p. 369 (1971)

4. Shame I H. : Mechanics of fluids, Third edition, Mc Graw-Hill $\operatorname{Inc}(1992)$

5. Sastry S.S.: Introductory Methods of Numerical Analysis, Third edition, Prentice. Hall of India Pt. New Delhi (2000). 